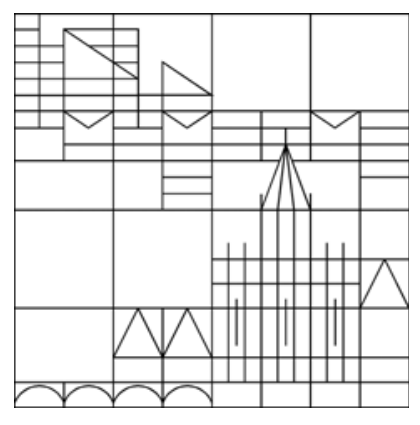

Symbolic-Numeric Computation of the Bernstein Coefficients of a Polynomial from Those of One of Its Partial Derivatives and of the Product of Two Polynomials

\author{
Jihad Titi \\ Jürgen Garloff
}

Konstanzer Schriften in Mathematik

Nr. 393, August 2020

ISSN $1430-3558$

Konstanzer Online-Publikations-System (KOPS)

URL: http://nbn-resolving.de/urn:nbn:de:bsz:352-2-1j0z.jyjdu0l3j8

(C) Fachbereich Mathematik und Statistik

Universität Konstanz

Fach D 197, 78457 Konstanz, Germany 



\title{
Symbolic-Numeric Computation of the Bernstein Coefficients of a Polynomial from Those of One of Its Partial Derivatives and of the Product of Two Polynomials
}

\author{
Jihad Titi $^{1}$ and Jürgen Garloff ${ }^{2,3}$ \\ ${ }^{1}$ Department of Applied Mathematics and Physics, Palestine Polytechnic University, \\ Hebron, Palestine \\ jihadtiti@yahoo.com \\ ${ }^{2}$ Department of Mathematics and Statistics, University of Konstanz, D-78464 \\ Konstanz, Germany \\ ${ }^{2}$ University of Applied Sciences / HTWG Konstanz, Institute for Applied Research, \\ Alfred-Wachtel-Str. 8, D-78462 Konstanz, Germany \\ juergen.garloff@htwg-konstanz.de
}

\begin{abstract}
The expansion of a given multivariate polynomial into Bernstein polynomials is considered. Matrix methods for the calculation of the Bernstein expansion of the product of two polynomials and of the Bernstein expansion of a polynomial from the expansion of one of its partial derivatives are provided which allow also a symbolic computation.
\end{abstract}

Keywords: Multivariate polynomial · Bernstein polynomial • Bernstein coefficient

MSC 2010: 65F30, 41-04

\section{Introduction}

In this paper, we consider the expansion of a multivariate polynomial into Bernstein polynomials over a box, i.e., an axis-aligned region, in $\mathbb{R}^{n}$. This expansion has many applications, e.g., in computer aided geometric design, robust control, global optimization, differerential and integral equations, finite element analysis [6]. A very useful property of this expansion is that the interval spanned by the minimum and maximum of the coefficients of this expansion, the so-called Bernstein coefficients, provides bounds for the range of the given polynomial over the considered box, see, e.g., $[8,10]$. A simple (but by no means economic) method for the computation of the Bernstein coefficients from the coefficients of the given polynomial is the use of formula (5) below. This formula (and also similar ones for the Bernstein coefficients over more general sets like sinplexes and polytopes) allows the symbolic computation of these quantities when the coefficients of the given polynomial depend on parameters. Some applications are making use of this symbolic computation: In [4, Sections 3.2 and 3.3], [5], see also the many references therein, the reachability computation and parameter synthesis with 
applications in biological modelling are considered. In [2,3], parametric polynomial inequalities over parametric boxes and polytopes are treated. Applications in static program analysis and optimization include dependence testing between references with linearized subscripts, dead code elimination of conditional statements, and estimation of memory requirements in the development of embedded systems. Applications which involve polynomials of higher degree or many variables require a computation of the Bernstein coefficients which is more economic than by formula (5). In [14] and [16], we have presented a matrix method for the computation of the Bernstein coefficients which is faster than the methods developed so far. In this paper, we consider firstly the case that we have already computed the Bernstein coefficients of two multivariate polynomials and wish to compute the Bernstein expansion of their product for which we present two approaches. For the univariate case see [7, Subsection 4.2]. Secondly, we show how the Bernstein coefficients of a multivariate polynomial can be computed from the Bernstein coefficients of its partial derivatives. This problem appears for example when bounds for the range of a complex polynomial over a rectangular region in the complex plane are wanted and the Cauchy-Riemann equations are employed, see [13, Section 4.3], [17], but it is also of interest by its own.

The organization of our paper is as follows. In the next section, we introduce the notation which is used throughout the paper. In Section 3, we first briefly recall the expansion of a multivariate real polynomial into Bernstein polynomials over a box and some of its fundamental properties. In the second part, we recall from $[14,16]$ a matrix method for the computation of the Bernstein coefficients. In Section 4 we present two matrix methods for the computation of the Bernstein coefficients of the product of two polynomials and in Section 5 the computation of the Bernstein coefficients of a polynomial from those of one of its partial derivatives.

\section{Notation}

In this section, we introduce the notation that we are using throughout this paper. Let $n \in \mathbb{N}$ (set of the nonnegative integers) be the number of variables. A multi-index $\left(i_{1}, \ldots, i_{n}\right) \in \mathbb{N}^{n}$ is abbreviated by $i$. In particular, we write 0 for $(0, \ldots, 0)$ and $e^{s}$ for the multi-index that has a 1 in position $s$ and 0's otherwise. Arithmetic operations with multi-indices are defined entrywise; the same applies to comparison between multi-indices. For the multi-index $i=\left(i_{1}, \ldots, i_{s}, \ldots, i_{n}\right)$ we define $i_{s, q}:=\left(i_{1}, \ldots, i_{s}+q, \ldots, i_{n}\right)$ and $i_{[s, q]}:=$ $\left(i_{1}, \ldots, q, \ldots, i_{n}\right), s \in\{1, \ldots, n\}, q \in \mathbb{Z}$. For $x=\left(x_{1}, \ldots, x_{n}\right) \in \mathbb{R}^{n}$, its monomials are defined as $x^{i}:=\prod_{s=1}^{n} x_{s}^{i_{s}}$. For $d=\left(d_{1}, \ldots, d_{n}\right) \in \mathbb{N}^{n}$ such that $i \leq d$, we use the compact notations $\sum_{i=0}^{d}:=\sum_{i_{1}=0}^{d_{1}} \ldots \sum_{i_{n}=0}^{d_{n}}$ and $\left(\begin{array}{l}d \\ i\end{array}\right):=\prod_{s=1}^{n}\left(\begin{array}{c}d_{s} \\ i_{s}\end{array}\right)$.

For the ease of presentation, we index all array entries starting from zero. 


\section{Bernstein Expansion}

\subsection{Bernstein Representation over the Unit Box}

In this section, we present fundamental properties of the Bernstein expansion over a box, e.g., [6, Subsection 5.1], [8,10], that are employed throughout the paper. For simplicity we consider the unit box $\boldsymbol{u}:=[0,1]^{n}$, since any compact nonempty box $\boldsymbol{x}$ of $\mathbb{R}^{n}$ can be mapped affinely onto $\boldsymbol{u}$. Let $\ell \in \mathbb{N}^{n}, a_{j} \in \mathbb{R}, j=$ $0, \ldots, \ell$, such that for $s=1, \ldots, n$

$$
\ell_{s}:=\max \left\{q \mid a_{j_{1}, \ldots, j_{s-1}, q, j_{s+1}, \ldots, j_{n}} \neq 0\right\} .
$$

Let $p$ be an $\ell$-th degree $n$-variate polynomial with the power representation

$$
p(x)=\sum_{j=0}^{\ell} a_{j} x^{j}
$$

We expand $p$ into Bernstein polynomials of degree $d, d \geq \ell$, over $\boldsymbol{u}$ as

$$
p(x)=\sum_{j=0}^{d} b_{j}^{(d)} B_{j}^{(d)}(x),
$$

where $B_{j}^{(d)}$ is the $j$-th Bernstein polynomial of degree $d$, defined as

$$
B_{j}^{(d)}(x):=\left(\begin{array}{c}
d \\
j
\end{array}\right) x^{j}(1-x)^{d-j},
$$

and $b_{j}^{(d)}$ is the $j$-th Bernstein coefficient of $p$ of degree $d$ over $\boldsymbol{u}$ which is given by

$$
b_{j}^{(d)}=\sum_{i=0}^{j} \frac{\left(\begin{array}{c}
j \\
i
\end{array}\right)}{\left(\begin{array}{c}
d \\
i
\end{array}\right)} a_{i}, \quad 0 \leq j \leq d,
$$

with the convention that $a_{i}:=0$ if $i \geq \ell, i \neq \ell$. We call (3) the Bernstein representation of $p$ and arrange the Bernstein coefficients in a multidimensional array $B(\boldsymbol{u})=\left(b_{j}^{(d)}\right)_{0 \leq j \leq d}$, the so-called Bernstein patch. Note that the Bernstein coefficients lying on the vertices of $B(\boldsymbol{u})$ are values of $p$ at the respective vertices of $\boldsymbol{u}$. More generally, the Bernstein coefficients on an $r$-dimensional face of $\boldsymbol{u}$, $r=0,1, \ldots, n-1$, are just the Bernstein coefficients lying on the respective faces of $B(\boldsymbol{u})[9$, Lemma 2]. E.g., assume that $\boldsymbol{v}$ is an $(n-1)$-dimensional face of $\boldsymbol{u}$ that is obtained by setting $x_{s}=0$ or 1 , for some $s \in\{1, \ldots, n\}$. For $i \in \mathbb{N}^{n}$ and $r \in \mathbb{N}$ we define

$$
i_{[s, r]}:=\left(i_{1}, \ldots, i_{s-1}, r, i_{s+1}, \ldots, i_{n}\right) .
$$

Then, the Bernstein coefficients of $p$ over $\boldsymbol{v}$ are given by

$$
b_{i}^{(d)}(p, \boldsymbol{v})= \begin{cases}b_{i_{[s, 0]},}, & \text { if } x_{s}=0 \\ b_{i_{\left[s, d_{s}\right]},}, & \text { if } x_{s}=1\end{cases}
$$




\subsection{Computation of the Bernstein Coefficients}

We recall from [16] a method for the computation of the Bernstein coefficients of the $n$-variate polynomial $p$ given in (2).

Matrix Method for the Unit Box The superscript $c$ denotes the cyclic ordering of the sequence of the indices, i.e., the order of the indices of the entries of the array under consideration is changed cyclically. This means that the index in the first position is replaced by the index in the second one, the index in the second position by the one in the third, .., the index in the $n$-th position by the one in the first position (see Figure 1 as an illustration in the trivariate case). So after $n$ cyclic orderings the sequence of the indices is again in its initial order. Note that in the bivariate case the cyclic ordering is just the usual matrix transposition.

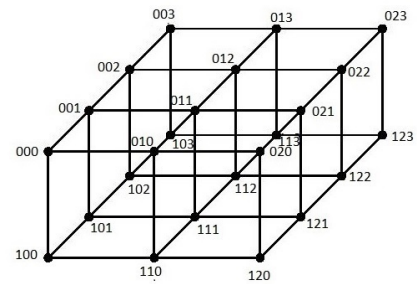

(a) $\Lambda(\boldsymbol{u})$

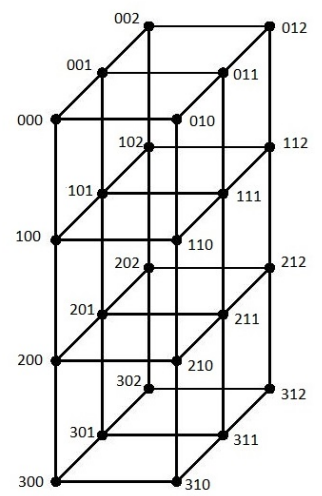

(c) $\left((\Lambda(\boldsymbol{u}))^{c}\right)^{c}$

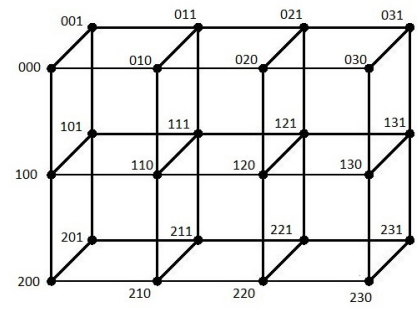

(b) $(\Lambda(\boldsymbol{u}))^{c}$

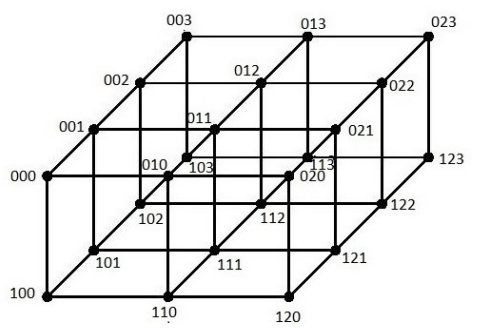

(d) $\left(\left((\Lambda(\boldsymbol{u}))^{c}\right)^{c}\right)^{c}$

Fig. 1: Cyclic ordering of a three-dimensional array with $\ell_{1}=1, \ell_{2}=2$, and $\ell_{3}=3$ 
The coefficients of $p$ are arranged in an $\left(\ell_{1}+1\right) \times \ell^{*}$ matrix $A$, where $\ell^{*}:=$ $\prod_{s=2}^{n}\left(\ell_{s}+1\right)$. The correspondence between the coefficients $a_{j}$ of $p$ and the entry

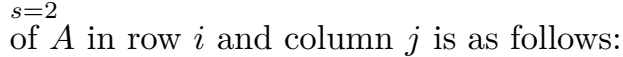

$$
\begin{aligned}
& i=j_{1}, \\
& j=j_{2}+\sum_{s=3}^{n} j_{s}\left(\ell_{2}+1\right) \cdot \ldots \cdot\left(\ell_{s-1}+1\right) .
\end{aligned}
$$

Then $A$ can be represented as the matrix

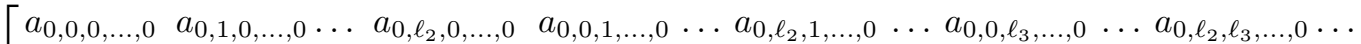

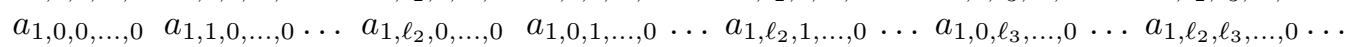

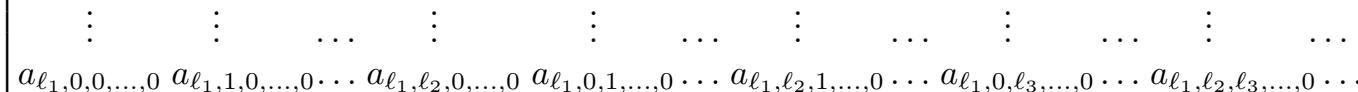

$$
\begin{aligned}
& \ldots a_{0,0, \ell_{3}, \ldots, \ell_{n}} \quad a_{0,1, \ell_{3}, \ldots, \ell_{n}} \ldots a_{0, \ell_{2}, \ell_{3}, \ldots, \ell_{n}} \\
& \ldots a_{1,0, \ell_{3}, \ldots, \ell_{n}} a_{1,1, \ell_{3}, \ldots, \ell_{n}} \ldots a_{1, \ell_{2}, \ell_{3}, \ldots, \ell_{n}} \\
& \left.\begin{array}{ccccc} 
& \vdots & \vdots & \ldots & \vdots \\
\ldots & a_{\ell_{1}, 0, \ell_{3}, \ldots, \ell_{n}} & a_{\ell_{1}, 1, \ell_{3}, \ldots, \ell_{n}} \ldots & a_{\ell_{1}, \ell_{2}, \ell_{3}, \ldots, \ell_{n}}
\end{array}\right] .
\end{aligned}
$$

The matrix $\Lambda(\boldsymbol{u})$ is obtained from $A$ by multiplying $a_{j}$ by $\left(\begin{array}{l}\ell \\ j\end{array}\right)^{-1}$. We put $\Lambda_{0}:=$ $\Lambda(\boldsymbol{u})$ and define for $s=1, \ldots, n$

$$
\Lambda_{s}:=\left(P_{s} \Lambda_{s-1}\right)^{c},
$$

where $P_{s}$ is the lower triangular Pascal matrix,

$$
\left(P_{s}\right)_{i j}:= \begin{cases}\left(\begin{array}{l}
i \\
j
\end{array}\right), & \text { if } j \leq i, \\
0, & \text { otherwise. }\end{cases}
$$

In (9), the matrix multiplication is performed according to the factorization, e.g., [1, Lemma 1],

$$
P_{s}=\prod_{\mu=1}^{\ell_{s}} K_{\mu}^{s}
$$

where the bidiagonal matrices $K_{\mu}^{s}, \mu=1, \ldots, \ell_{s}$, are given by

$$
\left(K_{\mu}^{s}\right)_{i j}:= \begin{cases}1, & \text { if } i=j \\ 1, & \text { if } i=j+1, \quad \ell_{s}-\mu \leq j \leq \ell_{s}-1, \\ 0, & \text { otherwise. }\end{cases}
$$


Define for $s=1, \ldots, n, r:=s \bmod n$. Then for $s=1, \ldots, n$ the entry in position $\left(v_{1}, v_{2}\right)$ in $\Lambda_{s-1}$ becomes $\left(v_{1}^{\prime}, v_{2}^{\prime}\right)$ in $\Lambda_{s}$, where

$$
\begin{aligned}
& v_{1}^{\prime}=v_{2} \bmod \left(\ell_{r+1}+1\right), \\
& v_{2}^{\prime}=\left\lfloor\frac{v_{2}}{\ell_{r+1}+1}\right\rfloor+v_{1} \prod_{\substack{m=1, m \neq s, r}}^{n}\left(\ell_{m}+1\right) .
\end{aligned}
$$

The Bernstein patch $B(\boldsymbol{u})$ arranged accordingly in the $\left(\ell_{1}+1\right) \times \ell^{*}$ Bernstein matrix, denoted by $\mathcal{B}(\boldsymbol{u})$, is given by $\Lambda_{n}$.

Matrix Method for a General Box Firstly, we affinely map a given box $\boldsymbol{x}$,

$$
\boldsymbol{x}=\left(\left[\underline{x}_{s}, \bar{x}_{s}\right]\right)_{s=1}^{n}, \text { with } \underline{x}_{s}<\bar{x}_{s}, s=1, \ldots, n,
$$

to the unit box $\boldsymbol{u}$ by

$$
z_{s}=\frac{x_{s}-\underline{x}_{s}}{\bar{x}_{s}-\underline{x}_{s}}, \quad s=1, \ldots, n \text {. }
$$

We sequentially transform $x_{s}, s=1, \ldots, n$. By substituting (14) in (2) for one $x_{s}, s=1, \ldots, n$, at a time, we obtain a polynomial $p^{\star}$ over $\boldsymbol{u}$. The coefficients of $p^{\star}$ arranged in an $\left(\ell_{1}+1\right) \times \ell^{\star}$ matrix, say $A^{\star}$, can be derived as follows from the matrix $A$ of the coefficients of $p$ given in (8): For $s=1, \ldots, n$ define

$$
Q_{s}:= \begin{cases}\tilde{D}_{s}\left(\frac{\bar{x}_{s}-\underline{x}_{s}}{\underline{x}_{s}}\right) P_{s}^{T} \tilde{D}_{s}\left(\underline{x}_{s}\right), & \underline{x}_{s} \neq 0, \\ \tilde{D}_{s}\left(\bar{x}_{s}\right), & \underline{x}_{s}=0,\end{cases}
$$

where $\tilde{D}_{s}(t)$ is the diagonal matrix of order $\ell_{s}+1$

$$
\tilde{D}_{s}(t):=\operatorname{diag}\left(1, t, t^{2}, \ldots, t^{\ell_{s}}\right), \quad s=1, \ldots, n .
$$

Then $A^{\star}$ can be represented as

$$
A^{\star}=\left(Q_{n}\left(\cdots\left(Q_{2}\left(Q_{1} A\right)^{c}\right)^{c} \cdots\right)^{c}\right)^{c} .
$$

By applying the procedure for the unit box to the matrix $A^{\star}$, we obtain the Bernstein patch of $p$ over $\boldsymbol{x}$.

Amount of Arithmetic Operations Assuming that $\kappa=\ell_{s}$ for all $s=$ $1, \ldots, n$, the presented matrix method requires $n \kappa \frac{(\kappa+1)^{n}}{2}$ additions and $n(\kappa+1)^{n}$ multiplications for the computation of the Bernstein coefficients over the unit box $\boldsymbol{u}$, and needs $n \kappa(\kappa+1)^{n}+n$ additions and $3 n(\kappa+1)^{n}+2 n(\kappa-1)+n$ multiplications for a general box. A verified version of this method which is taking into account of all rounding errors as well as data uncertainties was implemented by Dr. Florian Bünger, Hamburg University of Technology, Germany. It is included in the version 12 of the MATLAB toolbox INTLAB [11]. 


\section{Computation of the Bernstein Coefficients of the Product of Two Multivariate Polynomials}

Let $p$ and $q$ be two $n$-variate polynomials of degree $\ell(p)$ and $\ell(q)$, respectively, with the Bernstein expansions of degrees $d(p) \geq \ell(p)$ and $d(q) \geq \ell(q)$ over $\boldsymbol{x}$

$$
\begin{aligned}
& p(x)=\sum_{j=0}^{d(p)} b_{j}(p) B_{j}^{(d(p))}(x), \\
& q(x)=\sum_{i=0}^{d(q)} b_{i}(q) B_{i}^{(d(q))}(x) .
\end{aligned}
$$

For the ease of presentation we consider here only the unit box $\boldsymbol{u}$ and assume that $d(p)=\ell(p)$ and $d(q)=\ell(q)$. Then, the polynomial $p q$ resulting when multiplying $p$ and $q$ is of degree $\ell=\ell(p)+\ell(q)$. Hence, the Bernstein representation of $p q$ over $\boldsymbol{u}$ is given as

$$
p q(x)=\sum_{m=0}^{\ell} b_{m}(p q) B_{m}^{(\ell)}(x),
$$

where $b_{m}(p q)$ is the $m$-th Bernstein coefficient of $p q$ of degree $\ell$ over $\boldsymbol{u}, m=$ $0, \ldots, \ell$. Let $B(p, \boldsymbol{u}), B(q, \boldsymbol{u})$, and $B(p q, \boldsymbol{u})$ denote the Bernstein patches of $p, q$, and $p q$ over $\boldsymbol{u}$, respectively, and their corresponding Bernstein matrices are given by $\mathcal{B}(p, \boldsymbol{u}), \mathcal{B}(q, \boldsymbol{u})$ and $\mathcal{B}(p q, \boldsymbol{u})$. In this section, we present two matrix methods, which are named the first method and second method, for the computation of the Bernstein coefficients $b_{m}(p q)$ of $p q$.

\subsection{First Method}

By this method the Bernstein coefficients of $p q$ are computed from the Bernstein representation of $p$ and $q$. The representations (17a) and (17b) can be rewritten as

$$
\begin{aligned}
& p(x)=(1-x)^{\ell(p)} \sum_{j=0}^{\ell(p)} c_{j}(p)\left(\frac{x}{1-x}\right)^{j}, \\
& q(x)=(1-x)^{\ell(q)} \sum_{i=0}^{\ell(q)} c_{i}(q)\left(\frac{x}{1-x}\right)^{i},
\end{aligned}
$$

where $c_{j}(p)$ and $c_{i}(q)$ are called the scaled Bernstein coefficients of $p$ and $q$ which are given by

$$
\begin{aligned}
& c_{j}(p)=b_{j}(p)\left(\begin{array}{c}
\ell(p) \\
j
\end{array}\right), j=0, \ldots, \ell(p), \\
& c_{i}(q)=b_{i}(q)\left(\begin{array}{c}
\ell(q) \\
i
\end{array}\right), i=0, \ldots, \ell(q) .
\end{aligned}
$$


From (19a) and (19b), the power representation of $p q$ is obtained as

$$
p q(x)=(1-x)^{\ell} \sum_{m=0}^{\ell} c_{m}\left(\frac{x}{1-x}\right)^{m},
$$

where the $m$-th scaled Bernstein coefficient $c_{m}$ of $p q$ is given as

$$
c_{m}=\sum_{\mu=0}^{m} c_{\mu}(p) c_{m-\mu}(q), \quad m=0, \ldots, \ell
$$

with $c_{\mu}(p):=0$ if $\mu_{s}>\ell_{s}(p)$ and $c_{\mu}(q):=0$ if $\mu_{s}>\ell_{s}(q)$ for some $s \in\{1, \ldots, n\}$. Then, from (21) the $m$-th Bernstein coefficient of $p q$ is

$$
b_{m}(p q)=\frac{c_{m}}{\left(\begin{array}{c}
\ell \\
m
\end{array}\right)}, \quad 0 \leq m \leq \ell .
$$

In matrix language, the first method can be described as follows: Let for $s=$ $1, \ldots, n$, the diagonal matrix $D_{s}$ of order $\ell_{s}(p)+1$ be defined by

$$
D_{s}:=\operatorname{diag}\left(\left(\begin{array}{c}
\ell_{s}(p) \\
0
\end{array}\right),\left(\begin{array}{c}
\ell_{s}(p) \\
1
\end{array}\right), \ldots,\left(\begin{array}{l}
\ell_{s}(p) \\
\ell_{s}(p)
\end{array}\right)\right)
$$

and let $C(p)$ be the $\left(\ell_{1}(p)+1\right) \times \ell^{\star}(p)$ matrix, where $\ell^{\star}(p):=\prod_{s=2}^{n}\left(\ell_{s}(p)+1\right)$, which is obtained by

$$
C(p)=\left(D_{n}\left(\cdots\left(D_{2}\left(D_{1} \mathcal{B}(p, \boldsymbol{u})\right)^{c}\right)^{c} \cdots\right)^{c}\right)^{c} .
$$

It is easy to see that $(C(p))_{q_{1}, q_{2}}=c_{j}(p), q_{1}=0 \ldots \ell_{1}(p), q_{2}=0 \ldots, \ell^{\star}(p)-1$ and $j=0, \ldots, \ell(p)$, where the correspondence between the scaled Bernstein coefficient $c_{j}(p)$ and the entries of $C(p)$ can be determined by using (7).

Let us define for $s=1, \ldots, n, t=0, \ldots, \ell_{s}(q)$, the following $\left(\ell_{s}+1\right) \times\left(\ell_{s}(p)+\right.$ 1) matrices $W_{s}^{(t)}$ row-wise by

$$
\begin{aligned}
W_{s}^{(t)}[0, \ldots, t-1] & :=0, \\
W_{s}^{(t)}\left[t, \ldots, \ell_{s}(p)+t\right] & :=I_{\ell_{s}(p)+1}\left(\text { identity matrix of order } \ell_{s}(p)+1\right), \\
W_{s}^{(t)}\left[\ell_{s}(p)+t+1, \ldots, \ell_{s}\right] & :=0
\end{aligned}
$$

as a convention, we define for $t=0, \ell_{s}(q)$

$$
\begin{aligned}
W_{s}^{(t)}[0, \ldots,-1] & =\phi, \\
W_{s}^{(t)}\left[\ell_{s}+1, \ldots, \ell_{s}\right] & =\phi,
\end{aligned}
$$

where $\phi$ is a matrix of size $0 \times 0$. Assume that the scaled Bernstein coefficients of $p q$, see $(21)$, are arranged in the $\left(\ell_{1}+1\right) \times \ell^{\star}$ matrix $C(p q)$, with $\ell^{\star}=\prod_{s=2}^{n}\left(\ell_{s}+1\right)$ 
that is given by

$C(p q)=\sum_{i_{n}=0}^{\ell_{n}(q)} \cdots \sum_{i_{1}=0}^{\ell_{1}(q)}\left(W_{n}^{\left(i_{n}\right)}\left(\cdots\left(W_{2}^{\left(i_{2}\right)}\left(W_{1}^{\left(i_{1}\right)} c_{i_{1}, \ldots, i_{n}}(q) C(p)\right)^{c}\right)^{c} \cdots\right)^{c}\right)^{c}(25)$

such that $(C(p q))_{q_{1}, q_{2}}=c_{m}, q_{1}=0, \ldots, \ell_{1}, q_{2}=0, \ldots, \ell^{\star}-1$, and $m=$ $0, \ldots, \ell$. Here, the relation between the entries of $C(p q)$ and the scaled Bernstein coefficients of $p q, c_{m}, m=0, \ldots, \ell$, can be determined as in (7). From (25), we get

$$
\mathcal{B}(p q, \boldsymbol{u})=\left(D_{n}^{\prime}\left(\cdots\left(D_{2}^{\prime}\left(D_{1}^{\prime} C(p q)\right)^{c}\right)^{c} \cdots\right)^{c}\right)^{c}
$$

where for $s=1, \ldots, n, D_{s}^{\prime}$ is the inverse of the diagonal matrix $\operatorname{diag}\left(\left(\begin{array}{c}\ell_{s} \\ 0\end{array}\right),\left(\begin{array}{c}\ell_{s} \\ 1\end{array}\right), \ldots,\left(\begin{array}{c}\ell_{s} \\ \ell_{s}\end{array}\right)\right)$. A pseudocode for the first method is given in Algorithm 1. Its performance is illustrated in Appendix A.

\subsection{Second Method}

Let $a_{j}(p)$ and $a_{i}(q)$ be the $j$-th and the $i$-th coefficients of the power representations of $p$ and $q$, respectively, such that $j=0, \ldots, \ell(p)$ and $i=0, \ldots, \ell(q)$. Assume that $a_{j}(p)$ are arranged in an $\left(\ell_{1}(p)+1\right) \times \ell^{\star}(p)$ matrix $A(p)$. Recall that $p q$ is an $n$-variate polynomial of degree $\ell$. Then the power representation of $p q$ is given by

$$
p q(x)=\sum_{m=0}^{\ell} a_{m} x^{m}
$$

We arrange the coefficients of $p q$ in an $\left(\ell_{1}+1\right) \times \ell^{\star}$ matrix $\hat{A}$. In this method, the computation of the Bernstein coefficients of $p q$, see (18), is based on its power representation (27). The matrix description of this method is as follows: The entries of $\hat{A}$ are the entries of the matrix that is obtained from (25), where the $\left(r_{1}, r_{2}\right)$-th entry of $C(p)$ is replaced by the $\left(r_{1}, r_{2}\right)$-th entry of $A(p)$, where $r_{1}=0, \ldots \ell_{1}(p), r_{2}=0 \ldots, \ell^{\star}(p)-1$, and $c_{i}(q)$ is replaced by $a_{i}(q), 0 \leq i \leq \ell(q)$. Then the method presented in Subsection 3.2 is applied to compute the Bernstein coefficients of $p q$ starting from $\hat{A}$.

\subsection{Amount of Arithmetic Operations}

In Tables 1 and 2, the number of the arithmetic operations of both methods are presented. For simplicity, we assume that $\ell_{s}(p)=\ell_{s}(q)=\kappa$ for $s=1, \ldots, n$. Furthermore, we use the method from Subsection 3.2 for the computation of $B(p, \boldsymbol{u})$ and $B(q, \boldsymbol{u})$ in the first method, see $(24)$, and $B(p q, \boldsymbol{u})$ in the second method. 


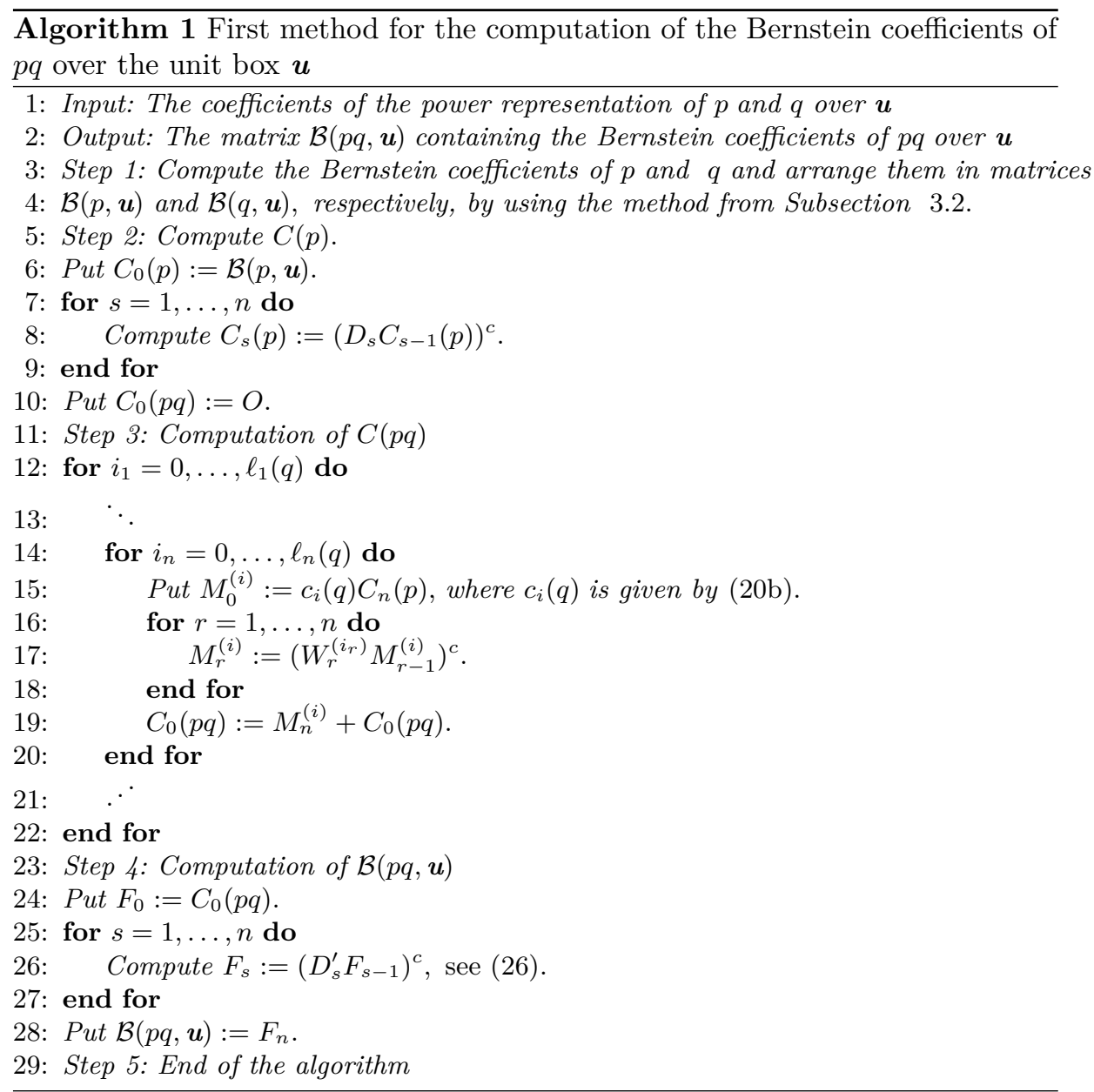

Table 1: Number of real arithmetic operations required to obtain $B(p q, \boldsymbol{u})$ by the first method

\begin{tabular}{|c|c|c|}
\hline Calculation of & number of additions & $\begin{array}{c}\text { number of } \\
\text { multiplications / divisions }\end{array}$ \\
\hline \hline $\begin{array}{c}B(p, \boldsymbol{u}) \text { and } B(q, \boldsymbol{u}) \text { by the } \\
\text { method in Subsection } 3.2\end{array}$ & $n \kappa(\kappa+1)^{n}$ & $2 n(\kappa+1)^{n}$ \\
\hline$C(p)$ & 0 & $(\kappa+1)^{2 n}$ \\
\hline$C(p q)$ & $\begin{array}{c}(\kappa+1)^{n}\left[(\kappa+1)^{n}-1\right]- \\
n \kappa(\kappa+1)^{n-1}\end{array}$ & $n(2 \kappa+1)^{n}$ \\
\hline$B(p q, \boldsymbol{u})$ & 0 & $(\kappa+1)$ \\
\hline
\end{tabular}


Table 2: Number of arithmetic operations to obtain $B(p q, \boldsymbol{u})$ by the second method

\begin{tabular}{|c|c|c|}
\hline Calculation of & number of additions & $\begin{array}{c}\text { number of } \\
\text { multiplications/divisions }\end{array}$ \\
\hline \hline$C(p q)$ & $\begin{array}{c}(\kappa+1)^{n}\left[(\kappa+1)^{n}-1\right]- \\
n \kappa(\kappa+1)^{n-1}\end{array}$ & $(\kappa+1)^{2 n}$ \\
\hline $\begin{array}{c}B(p q, \boldsymbol{u}) \text { by the method in } \\
\text { Subsection 3.2 }\end{array}$ & $n \kappa(2 \kappa+1)^{n}$ & $n(2 \kappa+1)^{n}$ \\
\hline
\end{tabular}

For the ease of comparison, we assume that the basis operations (addition, multiplication, and division) are taking the same time. Then we conclude that for $n \geq 4$ and $\kappa=1, n \geq 2$ and $\kappa=2,3$, and for all $n$ and $\kappa \geq 4$ the first method is superior to the second method. In addition, the first method has the advantage that all computations are performed $a b$ initio in the Bernstein representation such that the numerical stability of this representation with respect to perturbations of initial data, or rounding errors that occur during floating point calculations can be fully employed, see $[6$, Section 6$]$.

\section{$5 \quad$ Matrix Method for the Computation of the Bernstein Coefficients of a Multivariate Polynomial from Those of One of Its Partial Derivatives}

Let $p$ be an $n$-variate polynomial of degree $\ell$ with the power representation given as in (2). Assume that its coefficients are arranged in the matrix $A$, which is presented in (8). We expand $p$ into Bernstein polynomials of degree $d, d \geq \ell$, over the box $\boldsymbol{x}$ (13) as in (3). Without loss of generality, we assume that $d=\ell$. Recall that the Bernstein representation of the first partial derivative of $p$ with respect to $x_{s}, s \in\{1, \ldots, n\}$, is given by

$$
\frac{\partial p}{\partial x_{s}}=\sum_{i \leq \ell_{s,-1}} \tilde{b}_{i}^{\left(\ell_{s,-1}\right)} B_{i}^{\left(\ell_{s,-1}\right)}(x)
$$

where for $j=0, \ldots, \ell_{s,-1}$

$$
\tilde{b}_{j}^{\left(\ell_{s,-1}\right)}=\ell_{s}\left(b_{j_{s, 1}}^{(\ell)}-b_{j}^{(\ell)}\right)
$$

denotes the $j$-th Bernstein coefficient of $\frac{\partial p}{\partial x_{s}}$ of degree $\ell_{s,-1}$ over $\boldsymbol{x}$, i.e., the Bernstein coefficients of $\frac{\partial p}{\partial x_{s}}$ can be obtained from differences between its successive Bernstein coefficients, e.g., [9, formula (4)]. 
Assume that the Bernstein coefficients of $\frac{\partial p}{\partial x_{s}}, s \in\{1, \ldots, n\}$, are given and are arranged in the Bernstein patch $B\left(\frac{\partial p}{\partial x_{s}}, \boldsymbol{x}\right)$. In the following, we present a matrix method by which the Bernstein patch $B(p, \boldsymbol{x})$ that comprises the Bernstein coefficients of $p$ over $\boldsymbol{x}$ can be computed using $B\left(\frac{\partial p}{\partial x_{s}}, \boldsymbol{x}\right)$. Without loss of generality, we assume that $s=1$. From (29), it follows that

$$
b_{i_{1,1}}^{(\ell)}=\frac{\tilde{b}_{i}^{\left(\ell_{1,-1}\right)}}{\ell_{1}}+b_{i}^{(\ell)}, \quad i=0, \ldots, \ell_{1,-1} .
$$

In other words, for computing $B(p, \boldsymbol{x})$ it is sufficient to compute the Bernstein coefficients $b_{i_{[1,0]}}^{(\ell)}$, see $(6)$, then the remaining coefficients can be obtained iteratively using (30). The coefficients $b_{i_{[1,0]}}^{(\ell)}$ are the Bernstein coefficients of $p$ for $x_{1}=\underline{x}_{1}$. By the face value property of the Bernstein coefficients, see Subsection 3.1 , these coefficients are identical to those that are located at the corresponding $(n-1)$-dimensional face of $B(p, \boldsymbol{x})$; they are obtained from $B(p, \boldsymbol{x})$ by firstly freezing $p$ on the face of $\boldsymbol{x}$ with $x_{1}=\underline{x}_{1}$ and then computing the Bernstein coefficients of the resulting polynomial. In matrix language, the computation is as follows. Denote by $C_{1}$ the row vector of length $\ell^{\star}$, where $\ell^{\star}=\prod_{s=2}^{n}\left(\ell_{s}+1\right)$, that contains the coefficients of $p$ such that $x_{1}=\underline{x}_{1}$. For $\mu=1, \ldots, \ell_{1}$, we define the elementary bidiagonal matrices $H_{\mu}(x) \in \mathbb{R}^{\mu, \mu+1}$ by

$$
\left(H_{\mu}(x)\right)_{i, j}:= \begin{cases}1, & i=j, \\ x, & i=\mu, j=\mu+1, \\ 0, & \text { otherwise. }\end{cases}
$$

Then, $C_{1}$ can be obtained as

$$
C_{1}=H_{1}\left(\underline{x}_{1}\right) \cdots H_{\ell_{1}-1}\left(\underline{x}_{1}\right) H_{\ell_{1}}\left(\underline{x}_{1}\right) A .
$$

From $C_{1}$, we define the $\left(\ell_{2}+1\right) \times \prod_{r=3}^{n}\left(\ell_{r}+1\right)$ matrix $A_{1}$ with coefficients given for $q_{1}=0, \ldots, \ell_{2}$ and $q_{2}=0, \ldots, \prod_{r=3}^{n}\left(\ell_{r}+1\right)-1$ by

$$
\left(A_{1}\right)_{q_{1}, q_{2}}:=\left(C_{1}\right)_{q_{1}+q_{2}\left(\ell_{2}+1\right)+1} .
$$

Then the method from Subsection 3.2 for the computation of the Bernstein coefficients of $p$ on the face of $\boldsymbol{x}$ with $x_{1}=\underline{x}_{1}$ starting from $A_{1}$ is applied. We denote the resulting matrix by $B_{1}$ and arrange its entries in the row vector $C_{1}^{\prime}$ of length $\ell^{\star}$, such that for $r_{1}=1, \ldots, \ell^{\star}, v_{1}=0, \ldots, \ell_{2}$, and $v_{2}=0, \ldots, \prod_{r=3}^{n}\left(\ell_{r}+\right.$ 1) -1 , we have

$$
\left(C_{1}^{\prime}\right)_{r_{1}}=\left(B_{1}\right)_{v_{1}, v_{2}}
$$

where

$$
r_{1}=v_{1}+v_{2}\left(\ell_{2}+1\right)+1 \text {. }
$$


Let $B_{1}^{\prime}$ be the $\left(\ell_{1}+1\right) \times \ell^{\star}$ matrix defined for $r_{1}=0, \ldots, \ell_{1}$ and $r_{2}=$ $0, \ldots, \ell^{\star}-1$ by

$$
\left(B_{1}^{\prime}\right)_{r_{1}, r_{2}}= \begin{cases}\left(C_{1}^{\prime}\right)_{r_{2}+1}, & \text { if } r_{1}=0, \\ \left(\frac{1}{\ell_{1}} \mathcal{B}\left(\frac{\partial p}{\partial x_{s}}, \boldsymbol{x}\right)\right)_{r_{1}-1, r_{2}}, & \text { if } r_{1}=1, \ldots, \ell_{1} .\end{cases}
$$

For $\mu=1, \ldots, \ell_{1}$, we define the square matrices $H_{\mu}^{(1)}$ of order $\ell_{1}+1$, such that for $r_{v}=0, \ldots, \ell_{1}, v=1,2$, it is given by

$$
\left(H_{\mu}^{(1)}\right)_{r_{1}, r_{2}}:= \begin{cases}1, & r_{1}=r_{2} \\ 1, & r_{1}=\ell_{1}-\mu+1, r_{2}=\ell_{1}-\mu, \\ 0, & \text { otherwise. }\end{cases}
$$

Then, the Bernstein matrix $\mathcal{B}(p, \boldsymbol{x})$ that comprises the Bernstein coefficients of $p$ of degree $\ell$ over $\boldsymbol{x}$, can be calculated by

$$
\mathcal{B}(p, \boldsymbol{x})=H_{1}^{(1)} \cdots H_{\ell_{1}-1}^{(1)} H_{\ell_{1}}^{(1)} B_{1}^{\prime},
$$

where the correspondence between $\mathcal{B}(p, \boldsymbol{x})$ and $B(p, \boldsymbol{x})$ can be determined by using (7). As a consequence of our initial assumption, for computing $B(p, \boldsymbol{x})$ from $B\left(\frac{\partial p}{\partial x_{s}}, \boldsymbol{x}\right)$, where $s \in\{2, \ldots, n\}$, we firstly employ the cyclic ordering with respect to $x_{s}$, in such a way that the multiplications in (32) and (37) are well defined.

In Table 3, the number of arithmetic operations needed for the computation of $\mathcal{B}(p, \boldsymbol{x})$ is presented. For simplicity, we assume here that $\ell_{s}=\kappa, s=1, \ldots, n$.

Table 3: Number of real arithmetic operations required to obtain $\mathcal{B}(p, \boldsymbol{x})$ from one of the partial derivatives of $p$

\begin{tabular}{|c|c|c|}
\hline Calculation of & number of additions & $\begin{array}{c}\text { number of } \\
\text { multiplications } / \text { divisions }\end{array}$ \\
\hline \hline$C_{1}$ by (32) & $\kappa(\kappa+1)^{n-1}$ & $\kappa(\kappa+1)^{n-1}$ \\
\hline $\begin{array}{c}B_{1} \text { over a general box } \boldsymbol{x}^{\prime} \\
\text { which is obtained from } \boldsymbol{x} \text { by } \\
\text { freezing } x_{1}=\underline{x}_{1} \text { using the } \\
\text { method in Subsection } 3.2\end{array}$ & $(n-1) \kappa(\kappa+1)^{n-1}+n-1$ & $\begin{array}{c}3(n-1)(\kappa+1)^{n-1}+2(n- \\
1)(\kappa-1)+n-1\end{array}$ \\
\hline$B_{1}^{\prime}$ by $(35)$ & 0 & $\kappa(\kappa+1)^{n-1}$ \\
\hline $\mathcal{B}(p, \boldsymbol{x})$ by $(37)$ & $\kappa(\kappa+1)^{n-1}$ & 0 \\
\hline
\end{tabular}


In total, the computation of $\mathcal{B}(p, \boldsymbol{x})$ requires $(n+1) \kappa(\kappa+1)^{n-1}+n-1$ additions and $2 \kappa(\kappa+1)^{n-1}+3(n-1)(\kappa+1)^{n-1}+2(n-1)(\kappa-1)+n-1$ multiplications.

\section{References}

1. Alonso, P., Delgado, J., Gallego, R., Peña, J.M.: Conditioning and accurate computations with Pascal matrices. J. Comput. Appl. Math. 252, 21-26 (2013).

2. Clauss, P., Chupaeva, I.Yu.: Application of symbolic approach to the Bernstein expansion for program analysis and optimization. In: Duesterwald, E. (ed.) Compiler Construction. LNCS, vol. 2985, pp. 120-133, Springer, Berlin, Heidelberg, (2004).

3. Clauss, P., Fernández, F.J., Garbervetsky, D., Verdoolaege, S.: Symbolic polynomial maximization over convex sets and its application to memory requirement estimation, IEEE Trans. Very Large Scale Integration (VLSI) Systems 17 (8), 983-996 (2009).

4. Dang, T., Dreossi, T., Fanchon, É., Maler, O., Piazza, C., Rocca, A.: Set-based analysis for biological modelling. In: Liò, P., Zuliani, P. (eds.) Automated Reasoning for Systems Biology and Medicine, Series Computational Biology, vol. 30, pp. 157189, Springer Nature, (2019).

5. Dreossi, T.: Sapo: Reachability computation and parameter synthesis of polynomial dynamical systems. In: Proc. Int. Conf. Hybrid Systems: Computation and Control, pp. 29-34, ACM, New York, NY, (2017).

6. Farouki, R.T.: The Bernstein polynomial basis: A centennial retrospective. Comput. Aided Geom. Design 29, 379-419 (2012).

7. Farouki, R.T., Rajan, V.T.: Algorithms for polynomials in Bernstein form. Comput. Aided Geom. Design 5, 1-26 (1988).

8. Garloff, J.: Convergent bounds for the range of multivariate polynomials. In: Nickel, K. (ed.) Interval Mathematics 1985. LNCS, vol. 212, pp. 37-56. Springer, Berlin, Heidelberg (1986).

9. Garloff, J., Smith, A.P.: Solution of systems of polynomial equations by using Bernstein expansion. In: Alefeld, G., Rump, S., Rohn, J., and Yamamoto J. (eds.), Symbolic Algebraic Methods and Verification Methods, pp. 87-97. Springer (2001).

10. Rivlin, T.J.: Bounds on a polynomial. J. Res. Nat. Bur. Standards 74(B), 47-54 (1970).

11. Rump, S.M.: INTLAB-INTerval LABoratory. In: Csendes, T. (ed.) Developments in Reliable Computing, pp. 77-104. Kluwer Academic Publishers, Dordrecht (1999).

12. Smith, A.P.: Fast construction of constant bound functions for sparse polynomials, J. Global Optim. 43, 445-458 (2009).

13. Titi, J.: Matrix methods for the tensorial and simplicial Bernstein forms with application to global optimization, dissertation, University of Konstanz, Konstanz, Germany (2019).

14. Titi, J., Garloff, J.: Fast determination of the tensorial and simplicial Bernstein forms of multivariate polynomials and rational functions, Reliab. Comput. 25, 24-37 (2017).

15. Titi, J., Garloff, J.: Matrix methods for the simplicial Bernstein representation and for the evaluation of multivariate polynomials. Appl. Math. Comput. 315, 246-258 (2017).

16. Titi, J., Garloff, J.: Matrix methods for the tensorial Bernstein form. Appl. Math. Comput. 346, 254-271 (2019).

17. Titi, J., Garloff, J.: Bounds for the range of a complex polynomial over a rectangular region, submitted (2020). 


\section{A Example for the Performance of Algorithm 1}

Let $p$ and $q$ be bivariate polynomials of degree $(4,2)$ and $(3,2)$, respectively, with Bernstein matrices over $\boldsymbol{u}$

$$
\mathcal{B}(p, \boldsymbol{u})=\left[\begin{array}{ccc}
2 & 0 & -1 \\
-2 & 3 & 0 \\
1 & 3 & -3 \\
1 & 0 & 1 \\
0 & -1 & 0
\end{array}\right] \text { and } \mathcal{B}(q, \boldsymbol{u})=\left[\begin{array}{ccc}
-1 & -4 & -1 \\
2 & 0 & 5 \\
-2 & 3 & 0 \\
0 & 1 & 1
\end{array}\right]
$$

Then

$$
\begin{aligned}
& D_{1}=\operatorname{diag}(1,4,6,4,1) \text { and } D_{2}=\operatorname{diag}(1,2,1), \\
& C_{1}(p)=\left(D_{1} C_{0}(p)\right)^{c}=\left[\begin{array}{ccccc}
2 & -8 & 6 & 4 & 0 \\
0 & 12 & 18 & 0 & -1 \\
-1 & 0 & -18 & 4 & 0
\end{array}\right], C_{2}(p)=\left(D_{2} C_{1}(p)\right)^{c}=\left[\begin{array}{ccc}
2 & 0 & -1 \\
-8 & 24 & 0 \\
6 & 36 & -18 \\
4 & 0 & 4 \\
0 & -2 & 0
\end{array}\right] \text {; } \\
& M_{0}^{(1,1)}=M_{0}^{(2,2)}=M_{0}^{(3,0)}=O_{5,3}, \\
& M_{0}^{(0,0)}=\left[\begin{array}{ccc}
-2 & 0 & 1 \\
8 & -24 & 0 \\
-6 & -36 & 18 \\
-4 & 0 & -4 \\
0 & 2 & 0
\end{array}\right], M_{0}^{(0,1)}=\left[\begin{array}{ccc}
-16 & 0 & 8 \\
64 & -192 & 0 \\
-48 & -288 & 144 \\
-32 & 0 & -32 \\
0 & 16 & 0
\end{array}\right], M_{0}^{(0,2)}=\left[\begin{array}{ccc}
-2 & 0 & 1 \\
8 & -24 & 0 \\
-6 & -36 & 18 \\
-4 & 0 & -4 \\
0 & 2 & 0
\end{array}\right] \text {, } \\
& M_{0}^{(1,0)}=\left[\begin{array}{ccc}
12 & 0 & -6 \\
-48 & 144 & 0 \\
36 & 216 & -108 \\
24 & 0 & 24 \\
0 & -12 & 0
\end{array}\right], M_{0}^{(1,2)}=\left[\begin{array}{ccc}
30 & 0 & -15 \\
-120 & 360 & 0 \\
90 & 540 & -270 \\
60 & 0 & 60 \\
0 & -30 & 0
\end{array}\right], M_{0}^{(2,0)}=\left[\begin{array}{ccc}
-12 & 0 & 6 \\
48 & -144 & 0 \\
-36 & -216 & 108 \\
-24 & 0 & -24 \\
0 & 12 & 0
\end{array}\right] \text {, } \\
& M_{0}^{(2,1)}=\left[\begin{array}{ccc}
36 & 0 & -18 \\
-144 & 432 & 0 \\
108 & 648 & -324 \\
72 & 0 & 72 \\
0 & -36 & 0
\end{array}\right], M_{0}^{(3,1)}=\left[\begin{array}{ccc}
4 & 0 & -2 \\
-16 & 48 & 0 \\
12 & 72 & -36 \\
8 & 0 & 8 \\
0 & -4 & 0
\end{array}\right], M_{0}^{(3,2)}=\left[\begin{array}{ccc}
2 & 0 & -1 \\
-8 & 24 & 0 \\
6 & 36 & -18 \\
4 & 0 & 4 \\
0 & -2 & 0
\end{array}\right]
\end{aligned}
$$

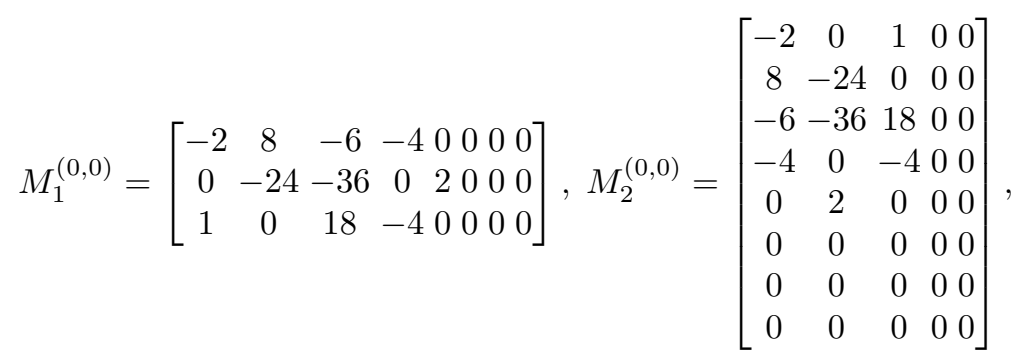




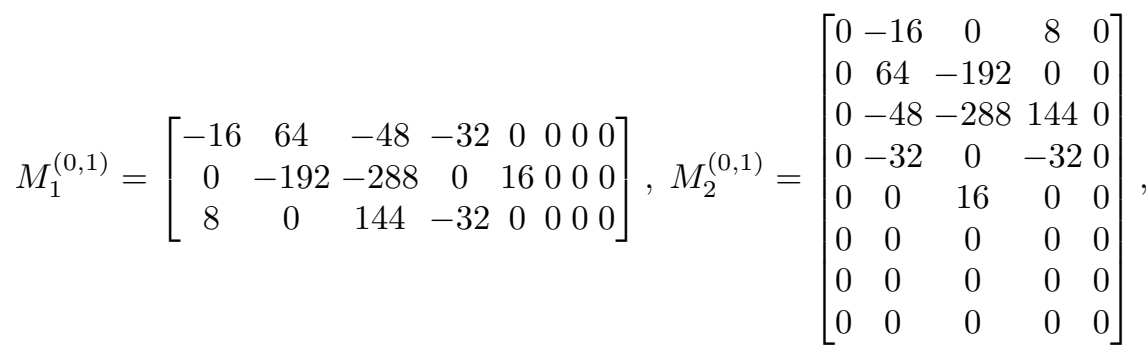

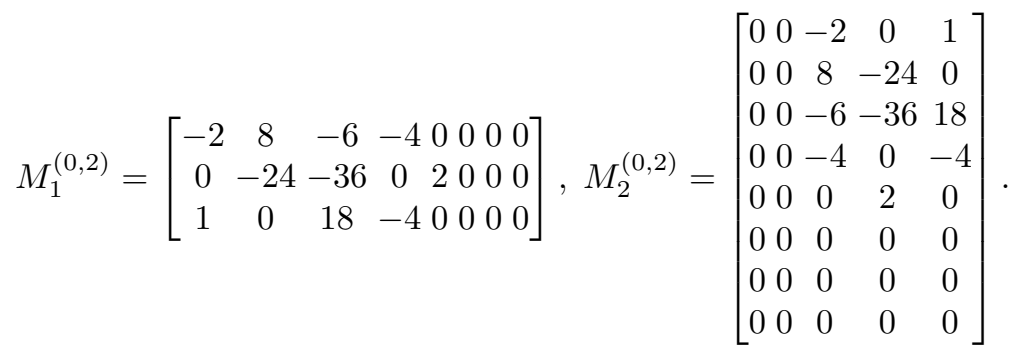

The remaining 18 matrices $M_{s}^{(i)}, s=1,2$, are formed analogously.

$$
\begin{gathered}
F_{0}=C_{0}(p q)=\left[\begin{array}{ccccc}
-2 & -16 & -1 & 8 & 1 \\
20 & 40 & -160 & -24 & -15 \\
-66 & 96 & -390 & 450 & 18 \\
80 & -100 & 408 & 506 & -275 \\
-12 & -122 & 896 & -298 & 60 \\
-24 & 72 & 54 & 42 & -18 \\
0 & 20 & -32 & 8 & 4 \\
0 & 0 & 4 & -2 & 0
\end{array}\right] ; \\
D_{1}^{\prime}=\operatorname{diag}\left(1, \frac{1}{7}, \frac{1}{21}, \frac{1}{35}, \frac{1}{35}, \frac{1}{21}, \frac{1}{7}, 1\right), D_{2}^{\prime}=\operatorname{diag}\left(1, \frac{1}{4}, \frac{1}{6}, \frac{1}{4}, 1\right) .
\end{gathered}
$$

Then the Bernstein matrix of the product of the polynomials $p$ and $q$ is given by

$$
\mathcal{B}(p q, \boldsymbol{u})=F_{2}=\left(D_{2}^{\prime}\left(D_{1}^{\prime} F_{0}\right)^{c}\right)^{c}=\left[\begin{array}{ccccc}
-2 & -4 & \frac{-1}{6} & 2 & 1 \\
\frac{20}{7} & \frac{10}{7} & \frac{-80}{21} & \frac{-6}{7} & \frac{-15}{7} \\
\frac{-22}{7} & \frac{8}{7} & \frac{-65}{21} & \frac{75}{14} & \frac{6}{7} \\
\frac{16}{7} & \frac{-5}{7} & \frac{68}{35} & \frac{253}{70} & \frac{-55}{7} \\
\frac{-12}{35} & \frac{-61}{70} & \frac{64}{15} & \frac{-149}{70} & \frac{12}{7} \\
\frac{-8}{7} & \frac{6}{7} & \frac{3}{7} & \frac{1}{2} & \frac{-6}{7} \\
0 & \frac{5}{7} & \frac{-16}{21} & \frac{2}{7} & \frac{4}{7} \\
0 & 0 & \frac{2}{3} & \frac{-1}{2} & 0
\end{array}\right] .
$$




\section{B Example for the Performance of the Method Presented in Section 5}

Let $p\left(x_{1}, x_{2}\right)=-504 x_{1}^{4} x_{2}^{2}-84 x_{1}^{4} x_{2}+288 x_{1}^{4}+6 x_{1}^{3} x_{2}^{2}+30 x_{1}^{3} x_{2}-60 x_{1}^{3}+36 x_{1}^{2} x_{2}^{2}-$ $20 x_{1}^{2} x_{2}+28 x_{1}^{2}-54 x_{1} x_{2}^{2}+21 x_{1} x_{2}-24 x_{1}+24 x_{2}^{2}-24 x_{2}+48$. Then

$$
\begin{aligned}
& \mathcal{B}\left(\frac{\partial p}{\partial x_{1}}, \boldsymbol{u}\right)=\left[\begin{array}{ccc}
-24 & \frac{-27}{2} & -57 \\
-4 & \frac{-9}{6} & \frac{-83}{3} \\
\frac{-140}{3} & \frac{-207}{6} & \frac{-67}{6} \\
1004 & \frac{-1743}{2} & -1241
\end{array}\right] \text { and } C_{1}^{\prime}=\mathcal{B}\left(p\left(0, x_{2}\right),[0,1]\right)=\left[\begin{array}{lll}
48 & 36 & 48
\end{array}\right] \\
& B_{1}^{\prime}=\left[\begin{array}{ccc}
48 & 36 & 48 \\
-6 & \frac{-27}{8} & \frac{-57}{4} \\
\frac{-4}{3} & \frac{-9}{24} & \frac{-83}{12} \\
\frac{-140}{12} & \frac{-207}{24} & \frac{-67}{12} \\
251 & \frac{-1743}{8} & \frac{-1241}{4}
\end{array}\right] \text {. }
\end{aligned}
$$

The Bernstein coefficients of $p$ over $\boldsymbol{u}$ are obtained from

$$
\begin{aligned}
& \mathcal{B}(p, \boldsymbol{u})=H_{1} H_{2} H_{3} H_{4} B_{1}^{\prime}
\end{aligned}
$$

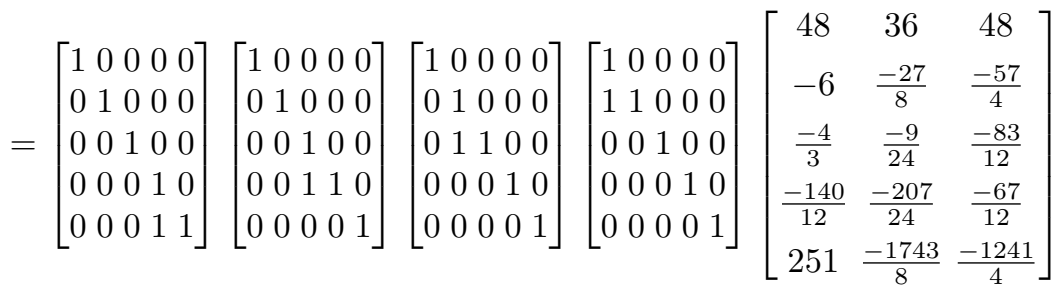

$$
\begin{aligned}
& =\left[\begin{array}{ccc}
48 & 36 & 48 \\
42 & \frac{261}{8} & \frac{135}{4} \\
\frac{122}{3} & \frac{774}{24} & \frac{322}{12} \\
29 & \frac{567}{24} & \frac{255}{12} \\
280 & \frac{483}{2} & -289
\end{array}\right]
\end{aligned}
$$

